\title{
Trial history biases the spatial programming of antisaccades
}

\author{
Tara Rastgardani • Victor Lau • Jason J. S. Barton • \\ Mathias Abegg
}

Received: 25 February 2011 / Accepted: 20 July 2012 / Published online: 5 September 2012

(c) Springer-Verlag 2012

\begin{abstract}
The historical context in which saccades are made influences their latency and error rates, but less is known about how context influences their spatial parameters. We recently described a novel spatial bias for antisaccades, in which the endpoints of these responses deviate towards alternative goal locations used in the same experimental block, and showed that expectancy (prior probability) is at least partly responsible for this 'alternategoal bias'. In this report we asked whether trial history also plays a role. Subjects performed antisaccades to a stimulus randomly located on the horizontal meridian, on a $40^{\circ}$ angle downwards from the horizontal meridian, or on a $40^{\circ}$ upward angle, with all three locations equally probable on any given trial. We found that the endpoints of antisaccades were significantly displaced towards the goal location of not only the immediately preceding trial $(n-1)$ but also the penultimate $(n-2)$ trial. Furthermore, this bias was mainly present for antisaccades with a short latency of $<250 \mathrm{~ms}$ and was rapidly corrected by secondary saccades. We conclude that the location of recent antisaccades biases the spatial programming of upcoming antisaccades,
\end{abstract}

T. Rastgardani · V. Lau · J. J. S. Barton

Departments of Medicine (Neurology), Ophthalmology and

Visual Sciences, Psychology, University of British Columbia,

Vancouver, Canada

T. Rastgardani

Faculty of Medicine, University of Toronto, Toronto, Canada

V. Lau

Faculty of Medicine, Griffith University, Brisbane, Australia

M. Abegg ( $\square)$

Universitätsklinik für Augenheilkunde Inselspital,

University of Bern, 3010 Bern, Switzerland

e-mail: mhabegg@hispeed.ch that this historical effect persists over many seconds, and that it influences mainly rapidly generated eye movements. Because corrective saccades eliminate the historical bias, we suggest that the bias arises in processes generating the response vector, rather than processes generating the perceptual estimate of goal location.

Keywords Antisaccade - Global effect - Range effect . Expectation

\section{Introduction}

Our current behaviour is influenced by what we were doing in the past and by what we expect to do in the future. Saccadic performance, for example, reflects not only the demands and properties of the task and stimulus in the current trial, but also the context in which they are executed. In particular, error rates and reaction times of a given trial may be influenced by properties of other trials performed the same experimental block (Fecteau and Munoz 2003; Tatler and Hutton 2007). These behavioural effects can have physiological correlates such as altered pre-target baseline activity in monkey collicular neurons (Dorris et al. 2000) and changes in human frontal eye field activity on functional neuroimaging (Manoach et al. 2007). The fact that performance on a given task is changed by the context - that is the past and the future - of a trial is important. First, the careful study of context in eye movements may illuminate some of the complexities of behaviour in real-life situations, in which stimuli and actions are embedded in a continuous stream of experience, with rich variability in the type of historical context and the degree of predictability of upcoming events. Second, contextual influences may reveal new aspects about the physiology of modulatory mechanisms involved in eye movements, and how they 
might use history and prediction to optimize responses to events. Finally, and pragmatically, experimental designs may need to consider or control for potential confounds from context that may obscure other results.

Not only the temporal dynamics of saccade generation depend on context. In a recent study we demonstrated that context can also affect the spatial programming of antisaccades. Antisaccades are eye movements that require a subject to look away from rather than towards a suddenly appearing stimulus (Hallett 1978). Compared to prosaccades, antisaccades are associated with less spiking activity in key ocular motor structures like the superior colliculus and frontal eye field in monkeys (Everling et al. 1999; Everling and Munoz 2000). If context also induces regions of weak spiking activity in a spatial map in those areas, such activity peaks might sum with saccade-related activity peaks to result in a weighted-average vector with slightly altered spatial coordinates, resulting in a deviation of saccade endpoint. As antisaccades have lower spiking activity peaks, this weighted-average shifting of saccade endpoint might be more evident for antisaccades than for prosaccades. Another reason for greater susceptibility of antisaccades to spatial biasing by context might be the larger cerebral network involved in generating antisaccades than in prosaccades (see Ettinger et al. 2008), which might create more opportunities for interactions with other cognitive operations.

Our previous study demonstrated that, when a subject performs antisaccades to one of two possible goal locations within an experimental block, the spatial positions of antisaccades directed to one goal location are deviated towards the location of the other goal (Abegg et al. 2010), an effect not seen with prosaccades. There are at least two reasons why such a spatial bias could occur. First, since the subject has recently been performing antisaccades to the other goal location in the experiment, a historical bias could arise. That is, a subject's saccade to the goal on the current trial might deviate slightly towards the other goal because they had recently made saccades to the other goal during preceding trials. Second, a spatial bias could arise from expectancy, or prior probability. That is, the fact that only two goal locations were used in an experimental block meant that subjects entered each trial with the expectation that the goal had a prior probability of 0.5 of occurring at either of those two locations, and 0 at all other locations. Targeting may then be directed at some combination of activity generated by expectancy at these locations with activity driven by appearance of the target.

Historical biases and expectancy biases can also be generated independent of each other. In a second experiment in our previous study (Abegg et al. 2010), we isolated the effects of expectancy. Instead of two locations we used three, one on, one above, and one below the horizontal meridian. For the goal on the horizontal meridian, there was no overall spatial bias from trial history when averaged over the entire experimental block, since there were equal numbers of trials with goals above and with goals below the meridian. To create an expectancy bias, we used cues on each trial that indicated that the goal could occur at one of two locations but not at the third. That is, they could occur on the horizontal meridian or above the meridian (but not below the meridian), or on the horizontal meridian or below the meridian (but not above the meridian). We found that these expectations alone were sufficient to bias horizontal antisaccades towards the other potential goal location, and away from the location that had a prior probability of zero.

The effects of expectancy alone in this second experiment were weaker than the alternate-goal bias in the original experiment, in which expectancy and historical effects may have been present, suggesting that factors other than expectancy may also contribute to the spatial biasing of antisaccades. In the current study we explore the possibility that trial history may be such a factor. The effect of trial history is here defined and measured as the influence of preceding trials on a current trial, even though these preceding trials do not affect the prior probability of an event in the current trial. We hypothesized that contextual biases could arise if spatial patterns of activity from recently executed antisaccades persist from one trial into the next. The potential for spatially specific effects to persist between trials is supported by other studies showing that the direction of a saccade in the previous trial affects the latency of saccades in the next trial (Barton et al. 2006; Reuter et al. 2006), an effect associated with altered pre-target activity in neurons in the superior colliculus in monkeys (Dorris et al. 2000). To determine whether trial history alone can generate spatial biases as well as these previously described temporal changes, we used an uncued version of the blocks in the second experiment of our prior study (Abegg et al. 2010). Without any cue, there is an equal prior probability on each trial of a goal occurring above, on, or below the horizontal meridian, so that saccades to the goal on the meridian cannot be biased towards the upper or lower field on the basis of expectancy in that trial. The effects of trial history were examined by determining the effects of the goal in the trials immediately preceding the current trial. If history alone can generate a spatial bias, then we should find that antisaccades are deviated towards the goal location of antisaccades in recent trials.

\section{Methods}

Subjects

Ten subjects of mean age 22.4 years (range, 18-35) participated, 2 males and 8 females. All subjects had normal or 
corrected-to-normal vision. The protocol was approved by the institutional review boards of Vancouver General Hospital and the University of British Columbia, and all subjects gave informed consent in accordance with the declaration of Helsinki.

\section{Apparatus and procedure}

Subjects were seated $57 \mathrm{~cm}$ away from a $21^{\prime \prime}$ monitor with dimensions of 1,024 pixels by 768 pixels. Head position was stabilized by forehead and chin rests. Lighting conditions in the room were kept constant across all subjects. Stimuli were created and presented on the monitor using SR Research Experiment Builder 1.1.2. Eye movements were recorded using the Eyelink 1000 system (SR Research Ltd., Mississauga, Canada). All subjects were calibrated with a nine-point array.

Subjects were instructed to look as accurately as possible to the equidistant location $180^{\circ}$ opposite to the direction of the stimulus. This study used antisaccades because our prior experiments found an alternate-goal bias for antisaccades only, not for prosaccades (Abegg et al. 2010). Each trial began with a black fixation cross that was centred on a white background. After 1,250 ms, the fixation cross disappeared and a stimulus appeared simultaneously. The stimulus was always a solid black disc of about $1^{\circ}$ in diameter, located at an eccentricity of $9.5^{\circ}$ of visual angle from central fixation point. The stimulus remained on the screen for $850 \mathrm{~ms}$ after the subject performed a saccade $>1.5^{\circ}$ in amplitude. The stimulus was then replaced by the display with the black fixation cross for the beginning of the next trial. Subjects were instructed to keep their gaze on the saccadic goal until the fixation cross reappeared at the screen centre.

Within each block there were three stimulus angular directions at equal distance from the screen centre, that is, 9.5 $5^{\circ}$ Angular directions were on $\left(0^{\circ}\right), 40^{\circ}$ above, or $40^{\circ}$ below the horizontal meridian. We chose $40^{\circ}$ as the angular difference because our prior study showed, first, that the spatial bias was still robust for this value and, second, that there were few if any responses misdirected to one of the other two goal locations with this degree of separation.

The experiment consisted of ten blocks of 93 trials each. Within each block stimuli were confined to a single hemifield, with five blocks for the right and five blocks for the left hemifield. Both block order and the trial order within each block were randomized.

Subjects were given both written and verbal instructions at the beginning of the experiment, outlining all possible stimuli and the appropriate antisaccade responses. Two practice blocks of nine trials each (one block for each hemifield) were performed before the ten experimental blocks.
Analysis

Data were analysed with SR Research Data Viewer 1.7.5. Saccades were detected when eye velocity exceeded $31 \%$ sec, acceleration exceeded $9,100 \% \mathrm{sec}^{2}$, and position changed by more than $1.5^{\circ}$. The first eye movement after stimulus onset that met these criteria was considered the saccadic response. Saccade latency was calculated as the time from target onset to saccadic onset. Saccades with latencies $<80 \mathrm{~ms}$ were considered anticipatory saccades, and those with latencies more than $800 \mathrm{~ms}$ were considered excessively delayed movements: both were excluded from further analysis. We also excluded trials in which the first saccade started from a point $>2^{\circ}$ from the central fixation point, and those with reflexive prosaccade-like errors in which the saccade vector was $>90^{\circ}$ away from the goal vector. On the basis of these criteria, $22 \%$ of all trials were excluded (range, 7-39\%).

Analysis was limited to trials with goals on the horizontal meridian, because only those trials had a bidirectional history, that is, had preceding trials pointing either up or down. The dependent variable was the vertical endpoint of the first saccadic response, which is simply the vertical position at the end of the saccade: given that the goal is on the horizontal meridian, this should not be significantly different from zero if there is no bias. Data were collapsed across the left and right hemifields and presented in graphs as though all trials were directed into the right hemifield. Values are reported as mean \pm standard error of the mean if not indicated otherwise. Other saccadic parameters including amplitude, latency, and proportion of directional errors are shown in Table 1.

Statistical analyses were performed in JMP 7.0 (www. jmp.com). In our primary analysis we categorized horizontal trials by the goal locations in the preceding trials. To examine the effects of both the immediately preceding $(n-1)$ trial and the penultimate $(n-2)$ trial, we included only correct trials that had been preceded by two consecutive correct trials. Thus, $49 \%$ of all possible trials were included on the basis of error-free sequences of at least three trials (range, 23-65\%). We subjected the means obtained for each subject to an analysis of variance (ANOVA) with main factors of prior goal location (up, middle, and down) and historical position $(n-1, n-2)$ of the prior goal location, and subject as a random factor. When significant effects were found, we used Tukey's honestly significant difference (HSD) test at an alpha level of 0.05 to identify the responsible contrasts, which we then quantified using linear contrasts. In addition, a priori comparisons between prior goal locations for each historical position were examined using linear contrasts.

In our second analysis, we examined whether the historical effect varied with the latency of the current 
Table 1 Mean amplitudes, saccadic latencies, and directional error rates, with standard error, for saccades preceded by trials with goals in either the upper quadrant $(\mathrm{U})$, the horizontal meridian $(\mathrm{M})$, or the lower quadrant $(\mathrm{L})$, in either the prior trial $(n-1)$ or the penultimate trial $(n-2)$

\begin{tabular}{lcccccc}
\hline & $(n-1) \mathrm{L}$ & $(n-1) \mathrm{M}$ & $(n-1) \mathrm{U}$ & $(n-2) \mathrm{L}$ & $(n-2) \mathrm{M}$ & $(n-2) \mathrm{U}$ \\
\hline Amplitude $\left(^{\circ}\right)$ & $7.74 \pm 0.35$ & $8.11 \pm 0.36$ & $7.75 \pm 0.29$ & $7.69 \pm 0.37$ & $7.97 \pm 0.36$ & $7.85 \pm 0.29$ \\
Latency (ms) & $234 \pm 11$ & $224 \pm 13$ & $227 \pm 10$ & $230 \pm 11$ & $223 \pm 11$ & $229 \pm 11$ \\
Directional error (\%) & $21.4 \pm 2.5$ & $16.7 \pm 3.2$ & $20.4 \pm 2.7$ & $16 \pm 2.8$ & $15.3 \pm 2.6$ & $18.7 \pm 2.6$ \\
\hline
\end{tabular}

Directional error is defined here as the number of saccades whose direction is more than $90^{\circ}$ away from that of the goal location, divided by the total number of saccades made

antisaccade response. It may be that the increased processing time available when responses have a longer latency may afford more accurate computation of goal coordinates: it has been shown, for example, that the effect of distractors on both trajectory and saccadic endpoint is greater on prosaccades with shorter latencies (Van der Stigchel and Theeuwes 2005; Findlay and Blythe 2009). If so, the magnitude of historical bias may also differ between rapidly executed and more delayed antisaccades. To examine a potential latency effect, we arbitrarily divided antisaccades into a short- and a long-latency group, using $250 \mathrm{~ms}$ as the latency criterion. This criterion was selected for two reasons. First, in experimental blocks with antisaccades alone, the mean response latency is around $250 \mathrm{~ms}$ - for example, Evdokimidis et al. (1996), Weber et al. (1998), and Van Zoest et al. (2008): hence, using this will give us reasonably equivalent numbers of short- and long-latency antisaccades. Second, experiments using multiple stimuli or simultaneous distractors show that spatial biases are more evident in saccades with latencies $<250 \mathrm{~ms}$ (Van Zoest et al. 2004; Van der Stigchel and Theeuwes 2005). Of the total of 1,820 trials included in our $n-1$ analysis, 579 were short-latency saccades and 1,241 were long-latency antisaccades. In the $n-2$ data set with a total of 1,528 trials, 493 were long-latency saccades and 1,035 were short-latency saccades. The proportion of shortand long-latency antisaccades varied considerably between subjects (the proportion of all antisaccades that were shortlatency was on average $70 \%$ (range, 32-91\%).

We thus analysed the subject mean data using ANOVA with historical position ( $n-1, n-2)$, prior goal location (up, middle, and down), and current antisaccade latency (short and long) as main factors, with subject as a random effect, using Tukey's HSD test with an alpha level of 0.05 to investigate the origins of significant results. For the latter purpose we also performed two subset ANOVAs, one for short-latency saccades alone and one for long-latency saccades only, with historical position $(n-1, n-2)$ and prior goal location (up, middle, and down) as main factors, with subject as a random effect.

In a third analysis, we examined whether spatial biases were corrected spontaneously by secondary saccades made by subjects. Many trials were characterized by a second saccade made after a very brief interval that still landed in the vicinity of the antisaccade goal, followed by a long fixation, before returning to the fixation cross at screen centre for the next trial. We asked whether the vertical endpoints of the final fixation measured just before the subject returned to screen centre also showed a persistent spatial bias. If the biases persisted, it would mean that the historical bias arose in structures and processes involved in estimating goal position from perceptual data. If the position of the final fixation did not show the spatial bias, it would imply that the stimulus-response re-mapping procedure involved in generating the antisaccade goal had possessed veridical spatial perceptual information and that the bias arose in structures creating the initial saccade vector towards the goal. Therefore, we performed an identical analysis to the first analysis of $(n-1)$ and $(n-2)$ effects, but this time using the final fixation rather than antisaccade endpoint as the dependent variable.

\section{Results}

\section{Historical effect}

The ANOVA showed a main effect of prior goal location $(F(2,45)=17.29, p<0.0001)$. Tukey's HSD test showed a significant difference between prior goal locations in the upper field and those on the horizontal meridian $(F(1,45)=16.02, \quad p<0.0002)$ or in the lower field $(F(1,45)=32.86, \quad p<0.0001)$. Although the effect of prior goal location appears larger from the $(n-2)$ trial than from the $(n-1)$ trial, there was no main effect of historical position $(n-1, n-2)$ nor any interaction between prior goal location and historical position (Fig. 1, Table 2). A priori linear contrasts showed a difference between $(n-1)$ goal locations in the upper field and those on the horizontal meridian $(F(1,45)=4.10, p<0.049)$ and those in the lower field $(F(1,45)=6.45, p<0.015)$. Similarly, there were differences between $(n-2)$ goal locations in the upper field and those on the horizontal meridian $(F(1,45)=13.22, p<0.0007)$ and those in the lower field $(F(1,45)=30.99, p<0.0001)$. 


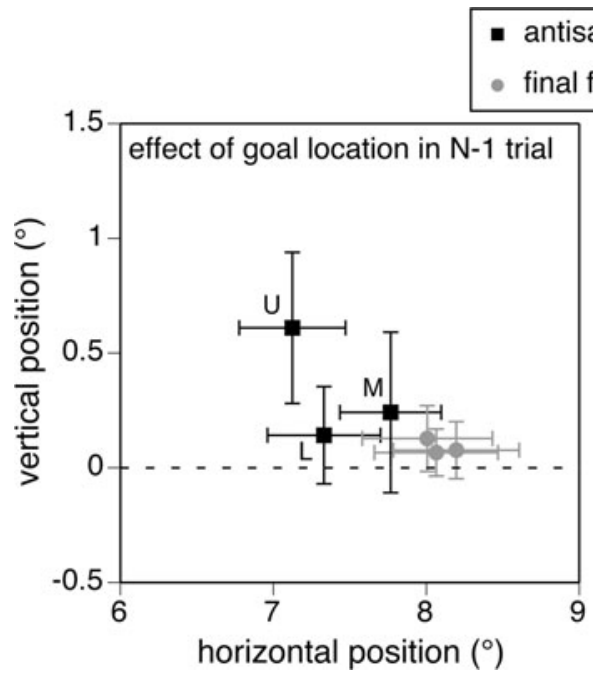

Fig. 1 Results for effects from the goal location in the immediately preceding $(n-1)$ trial (left graph) and in the penultimate $(n-2)$ trial (right graph). Data are for antisaccades from trials that had goals located on the horizontal meridian. In each graph, the mean endpoints of the first antisaccade response are shown as black squares. The mean final fixations at the end of the trial, after secondary corrective

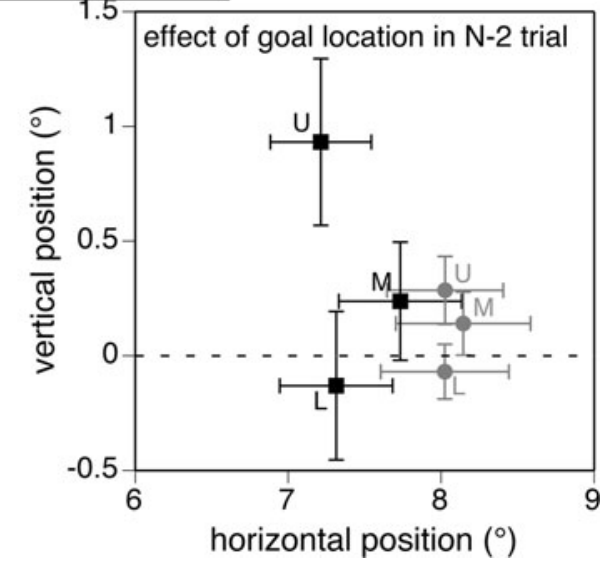

saccades, are shown as grey discs. ' $U$ ' indicates mean response from trials preceded by a trial with a goal in the upper quadrant, ' $L$ ' indicates those preceded by a trial with a goal in the lower quadrant, and ' $\mathrm{M}$ ' indicates those preceded by a trial with a goal on the horizontal meridian. Error bars indicate one standard error

Table 2 Vertical deviations

Positive values are upwards, negative are downwards $S E$ standard error

\begin{tabular}{|c|c|c|c|c|c|c|}
\hline \multirow{2}{*}{$\begin{array}{l}\text { Historical } \\
\text { position }\end{array}$} & \multicolumn{2}{|l|}{ Upper } & \multicolumn{2}{|c|}{ Horizontal } & \multicolumn{2}{|l|}{ Lower } \\
\hline & Mean & SE & Mean & SE & Mean & SE \\
\hline \multicolumn{7}{|c|}{ Analysis 1: vertical antisaccade endpoint } \\
\hline$n-1$ & 0.61 & 0.33 & 0.24 & 0.35 & 0.14 & 0.22 \\
\hline$n-2$ & 0.93 & 0.36 & 0.24 & 0.26 & -0.13 & 0.32 \\
\hline \multicolumn{7}{|c|}{ Analysis 2: endpoint for short- versus long-latency antisaccades } \\
\hline \multicolumn{7}{|c|}{ Short latency $<250 \mathrm{~ms}$} \\
\hline$n-1$ & 0.82 & 0.37 & 0.31 & 0.41 & 0.10 & 0.26 \\
\hline$n-2$ & 1.05 & 0.40 & 0.31 & 0.29 & -0.27 & 0.37 \\
\hline \multicolumn{7}{|c|}{ Long latency $>250 \mathrm{~ms}$} \\
\hline$n-1$ & 0.00 & 0.23 & 0.46 & 0.37 & 0.27 & 0.11 \\
\hline$n-2$ & 0.34 & 0.23 & 0.33 & 0.33 & 0.25 & 0.17 \\
\hline \multicolumn{7}{|c|}{ Analysis 3: final fixation } \\
\hline$n-1$ & 0.13 & 0.14 & 0.08 & 0.12 & 0.07 & 0.10 \\
\hline$n-2$ & 0.29 & 0.15 & 0.14 & 0.14 & -0.07 & 0.12 \\
\hline
\end{tabular}

goal locations for short-latency saccades $(F(1,90)=16.42$, $p<0.0001)$ but not for long-latency saccades.

To investigate this further, we performed subset ANOVAs for only short- or only long-latency antisaccades. The subset ANOVA for only short-latency saccades showed a main effect of prior goal location $(F(2,45)=$ $19.02, p<0.0001)$. Tukey's HSD test showed a difference between trials with a prior goal location in the upper field and those with a prior goal location either on the horizontal meridian $(F(1,45)=13.87, p<0.0005)$ or in the lower field $(F(1,45)=37.44, p<0.0001)$. There was no main effect of historical position nor any interaction between 


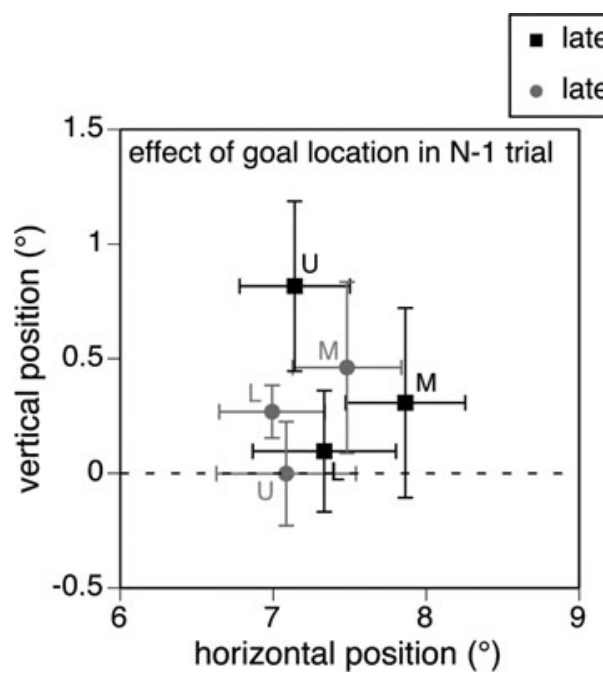

Fig. 2 Secondary analysis comparing short-latency $(<250 \mathrm{~ms})$ and long-latency $(>250 \mathrm{~ms})$ antisaccades, for the effect of goal location in the immediately preceding $(n-1)$ trial (left graph) and in the penultimate $(n-2)$ trial (right graph). Data are for antisaccades from trials that had goals located on the horizontal meridian. In each graph, the mean endpoints of short-latency antisaccades are shown as black

prior goal location and historical position. The subset ANOVA for long-latency saccades alone found no main effect of either prior goal location or historical position, nor any interaction between the two. Hence, the historical effect of prior goal location is seen only in short-latency antisaccades.

\section{Final fixation}

In this third analysis we asked whether the historical alternate-goal bias was still present in the position of the final fixation, often arrived at after secondary corrective saccades made by the subjects (Fig. 1, Table 2). The ANOVA showed a main effect for prior goal location $(F(2,45)=10.76, p<0.0002)$. While there was no main effect of historical position, there was a trend to an interaction between prior goal location and historical position $(F(2,45)=3.15, p=0.053)$. Tukey's HSD test showed that the only significant contrast was between $(n-2)$ upper and lower prior goal locations $(F(1,45)=25.09$, $p<0.0001)$. Of note $(n-1)$, goal locations had no effect on the position of the final fixation.

These data show that after corrective saccades, no significant bias remains from the prior goal location in the $(n-1)$ trial, but there is still an effect from the $(n-2)$ trial, though this appears smaller than the effect on antisaccade endpoint. To confirm that the effect of the prior goal location was reduced for final fixation compared to the antisaccade endpoint, we computed for each subject an index of historical bias, by subtracting the mean position following a trial with a goal in the lower quadrant from the mean position following a trial with goal in the upper quadrant. This was done for both antisaccade endpoint and final fixation (Fig. 3). We then compared the two for both the $(n-1)$ and the $(n-2)$ effect. The results showed significantly less historical bias for final fixation than for antisaccade endpoint, for both the $(n-1)(t(9)=2.31, p<0.047)$ and the $(n-2)$ historical positions $(t(9)=3.04, p<0.015)$. While the endpoint of the initial saccade showed both considerable variability and bias towards the preceding goal locations, the final fixation endpoint shows less bias and less variability (Fig. 3).

\section{Discussion}

While prior studies have shown how the context of other trials influences the latency and error rate of saccadic performance (Fecteau and Munoz 2003; Tatler and Hutton 2007), our results demonstrate that trial history influences not just when the upcoming trial is executed but also the spatial properties of the eye movement that is performed. We show that under experimental conditions where prior probability effects are balanced to minimize the effects of expectancy, there remains a significant bias of antisaccades to land slightly biased towards the goal of recently performed antisaccades. These historical effects are persistent, since they can be detected from not just the preceding trial but also the penultimate trial: hence, they persist over at 


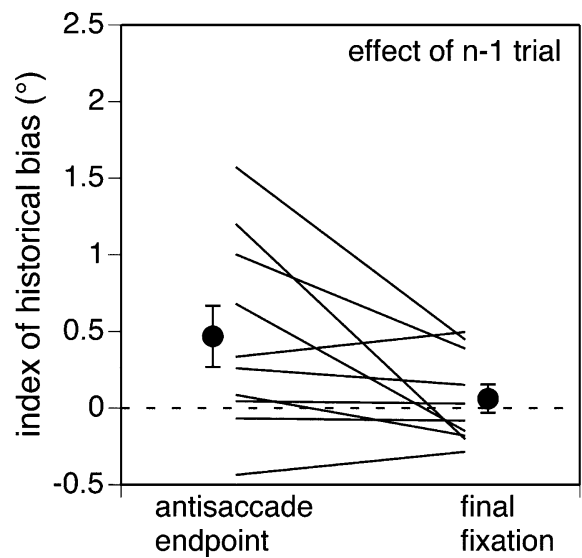

Fig. 3 Within-subject display of the reduction in the historical alternate-goal bias from the endpoint of the first antisaccade response to the final fixation after corrective saccades. The index is the mean for position data from antisaccades preceded by trials with goals in the upper hemifield, minus the mean from trials with goals in the

least several seconds. This historical effect complements our prior demonstration that expectancy can generate similar biases independent of effects based on trial history. Together, these two studies thus show that the phenomenon of alternate-goal bias has two components, one from the past (historical bias) and one concerning the immediate future (expectancy)

This alternate-goal bias has some similarities to the 'range effect' shown for prosaccade amplitude. In the best demonstration, prosaccades to targets of around $7^{\circ}-11^{\circ}$ eccentricity were hypometric if trials were in a block with other less eccentric targets, and hypermetric if the block had more eccentric targets (Kapoula 1985). This established that computation of saccadic amplitude is a dynamic product that can be modulated by other factors. As was the case with the first experiment of our original demonstration of alternate-goal bias (Abegg et al. 2010), the range effect potentially incorporates both expectancy and historical effects (He and Kowler 1989).

A second phenomenon of relevance to alternate-goal bias is the well-known global effect (Findlay 1982). In the global effect distractors are presented simultaneously with a stimulus for prosaccades. If presented within a range of angles $\left( \pm 20^{\circ}\right)$, a distractor affects saccadic amplitude even when the subject is told to ignore it, whereas a distractor outside this range slows saccadic latency (Walker et al. 1997; Findlay and Blythe 2009). The global effect biases prosaccades towards the distractor, just as our historical effect biases antisaccades towards the previous goal. The difference between the two, though, is that the global effect is generated by a simultaneous distractor on the current trial, while the historical effect is generated by the target and goal in preceding trials.

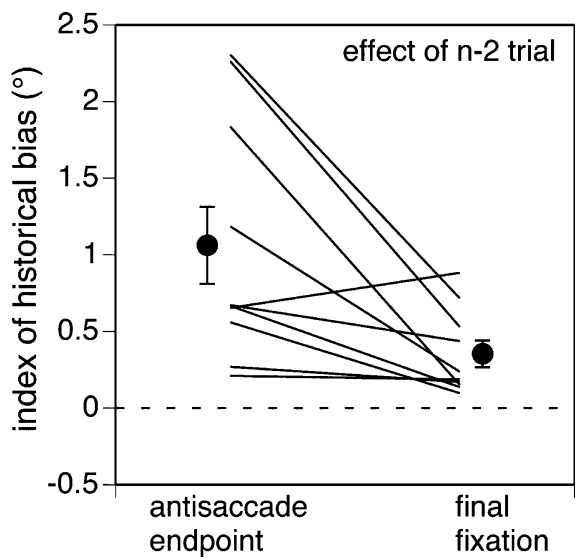

lower hemifield, calculated for each subject. Data for the effect of goal location in the immediately preceding $(n-1)$ trial are shown in the left graph, and for goal location in the penultimate $(n-2)$ trial in the right graph. Discs show group means, with error bars indicated one standard error

It has been proposed that the global effect reflects averaging of activity peaks from two different spatiotopic locations, one at the location of the stimulus and one at the location of the distractor (Van Opstal and Van Gisbergen 1990). Activity patterns consistent with this explanation have been found in monkey superior colliculus (Glimcher and Sparks 1993; Edelman and Keller 1998). In the case of the historical bias we demonstrate in this report, it may be that peaks of activity in prior trials have some temporal persistence and remain weakly evident in subsequent trials. Hence, we speculate that a historical bias may represent a weighted averaging of current and prior trial activity in some retinotopic map coding for saccade direction. Whether this averaging would occur in the superior colliculus or in some other ocular motor structure remains to be determined.

Our secondary analyses show findings that also provide two interesting behavioural parallels between the historical bias and the effects of simultaneous distractors. First, just as the global effect and distractor effects on trajectory are more pronounced for short-latency prosaccades (Van der Stigchel and Theeuwes 2005; Findlay and Blythe 2009), the historical bias we found in this report is more pronounced for antisaccades with latencies of $<250 \mathrm{~ms}$. Second, secondary saccades after the initial antisaccade tended to reduce alternate-goal bias in the final fixation position before termination of the trial. Again, similar reductions in spatial deviation have been shown for corrective secondary saccades in the global effect (Eggert et al. 2002).

One possible interpretation of these latter two results is that the activity persisting at the goal location from the prior trial rapidly decays after the appearance of the new goal location of the current trial. Indeed, in the case of 
simultaneous distractors, rapid decay of exogenous, stimulus-related influences has been advanced as the explanation for the greater impact of saliency and distractors on prosaccades with shorter latencies (Van Zoest et al. 2004). Models have accounted for the larger effect of distractors on short-latency saccades by suggesting that activity at the saccadic goal location generates lateral inhibition that quickly builds up, suppressing other locations, and thus causing a rapid decay in activity generated by distractors at other locations (Trappenberg et al. 2001). The result is a greater influence of exogenous stimulus-driven activity in short-latency responses and a greater influence of endogenous goal-driven activity in long-latency responses (Van Zoest et al. 2004). In our historical bias, the impact of the location of the prior trial might be viewed as a persistent influence from preceding activity, likewise extraneous to current goal-driven activity, which is therefore also maximal in short-latency antisaccades.

However, this explanation is not entirely consistent with the fact that we can still find an effect from the $(n-2)$ trial, which shows that alternate-goal bias persists for several seconds at least. Rather, these findings suggest that during the additional processing time available with a longlatency antisaccade, or in the interval between the first response and secondary corrective saccades, further goaldriven computations related to goal location are able to counteract the bias originating from prior goals, though they do not eliminate it from the system. That is, the influence of the prior goal location is persistent and does not rapidly decline; rather, within a trial it is the goaldriven influence that grows so that it dominates over the historical context effect in long-latency responses. Furthermore, the other implication of the reduction in the historical alternate-goal bias by secondary corrective saccades is that the ocular motor system retains accurate perceptual estimates of the true goal location: hence, the bias must originate in structures downstream of those areas generating these perceptual computations.

Finally, it must be stressed that, while the above inferences point to current goal programming rather than current stimulus processing as the site at which the bias is operating, our experiments do not clarify what property of the prior trial generates the bias, whether it is the saccadic response or its stimulus-related activity. Further studies dissociating saccadic and stimulus properties of the prior trials would be useful in addressing this point.

In summary, our work shows specifically that trial history alone can influence saccadic endpoints. Antisaccades were biased towards the goal locations of immediately preceding antisaccades, a type of 'immediate plasticity' (Dorris et al. 2000) that persisted over several seconds, from at least two trials before. This demonstrates the flexibility of saccadic behaviour and shows how our current responses to the environment can be shaped by the contingency of recent experience.

Acknowledgments This work was supported by CIHR operating grant MOP-81270. TR was supported by an AAN-SIGN summer fellowship grant, MA by a grant from the Schweizerische Stiftung für medizinisch-biologische Stipendien, and JB by a Canada Research Chair and a Senior Scholar award from the Michael Smith Foundation for Health Research. We thank Meinrad Abegg for his help with statistical analysis, and Wieske van Zoest and Stefan van der Stigchel for informative discussions and suggestions.

\section{References}

Abegg M, Rodriguez AR, Lee H, Barton JJS (2010) 'Alternate-goal bias' in antisaccades and the influence of expectation. Exp Brain Res 203:553-562

Barton JJS, Manoach D, Goff D (2006) The inter-trial effects of stimulus and saccadic direction on prosaccades and antisaccades, in controls and schizophrenic patients. Exp Brain Res 174:487-498

Dorris M, Paré M, Munoz D (2000) Immediate neural plasticity shapes motor performance. J Neurosci 20:RC52

Edelman JA, Keller EL (1998) Dependence on target configuration of express saccade-related activity in the primate superior colliculus. J Neurophysiol 80:1407-1426

Eggert T, Sailer U, Ditterich J, Straube A (2002) Differential effect of a distractor on primary saccades and perceptual localization. Vision Res 42:2969-2984

Ettinger U, Ffytche DH, Kumari V, Kathmann N, Reuter B, Zelaya F, Williams SC (2008) Decomposing the neural correlates of antisaccade eye movements using event-related FMRI. Cereb Cortex 18(5):1148-1159

Evdokimidis I, Constantinidis T, Liakopoulos D, Papageorgiou C (1996) The increased reaction time of antisaccades: what makes the difference? Int J Psychophysiol 22:61-65

Everling S, Munoz DP (2000) Neuronal correlates for preparatory set associated with pro-saccades and anti-saccades in the primate frontal eye field. J Neuriosci 20:387-400

Everling S, Dorris MC, Klein RM, Munoz DP (1999) Role of primate superior colliculus in preparation and execution of anti-saccades and pro-saccades. J Neurosci 19:2740-2754

Fecteau J, Munoz D (2003) Exploring the consequences of the previous trial. Nat Rev Neurosci 4:435-443

Findlay JM (1982) Global processing for saccadic eye movements. Vision Res 22:1033-1045

Findlay JM, Blythe HI (2009) Saccade target selection: do distractors affect saccade accuracy? Vision Res 49:1267-1274

Glimcher P, Sparks D (1993) Representation of averaging saccades in the superior colliculus of the monkey. Exp Brain Res 95: 429-435

Hallett P (1978) Primary and secondary saccades to goals defined by instructions. Vis Res 18:1279-1296

He P, Kowler E (1989) The role of location probability in the programming of saccades: implications for "center-of-gravity" tendencies. Vis Res 29:1165-1181

Kapoula Z (1985) Evidence for a range effect in the saccadic system. Vis Res 25:1155-1157

Manoach DS, Thakkar KN, Cain MS, Polli FE, Edelman JA, Fischl B, Barton JJ (2007) Neural activity is modulated by trial history: a functional magnetic resonance imaging study of the effects of a previous antisaccade. J Neurosci 27:1791-1798

Reuter B, Philipp A, Koch I, Kathmann N (2006) Effects of switching between leftward and rightward pro-and antisaccades. Biol Psychol 40:122-130 
Tatler BW, Hutton SB (2007) Trial by trial effects in the antisaccade task. Exp Brain Res 179:387-396

Trappenberg TP, Dorris MC, Munoz DP, Klein RM (2001) A model of saccade initiation based on the competitive integration of exogenous and endogenous signals in the superior colliculus. J Cogn Neurosci 13:256-271

Van der Stigchel S, Theeuwes J (2005) Relation between saccade trajectories and spatial distractor locations. Brain Res Cogn Brain Res 25:579-582

Van Opstal A, Van Gisbergen JAM (1990) Role of monkey superior colliculus in saccade averaging. Exp Brain Res 79: $143-149$
Van Zoest W, Donk M, Theeuwes J (2004) The role of stimulusdriven and goal-driven control in saccadic visual selection. J Exp Psychol Hum Percept Perform 30:746-759

Van Zoest W, Van der Stigchel S, Barton JJS (2008) Distractor effects on saccade trajectories: a comparison of prosaccades, antisaccades, and memory-guided saccades. Exp Brain Res 186:431-442

Walker R, Deubel H, Schneider WX, Findlay JM (1997) Effect of remote distractor on saccade programming: evidence for an extended fixation zone. J Neurophysiol 78:1108-1119

Weber H, Durr N, Fischer B (1998) Effects of pre-cues on voluntary and reflexive saccade generation. II. Pro-cues for anti-saccades. Exp Brain Res 120:417-431 